\section{A rare cause of acute abdominal pain}

\author{
Viviana Bozzano, ${ }^{1}$ Paolo Aseni, ${ }^{2}$ \\ Sandro Di Domenico, ${ }^{2}$ \\ Riccardo Colombo, ${ }^{2}$ Mariella Corciulo, ${ }^{2}$ \\ Andrea Bellone ${ }^{2}$ \\ 'Department of Internal Medicine, \\ Fondazione IRCCS Ca' Granda, Ospedale \\ Maggiore Policlinico, University of Milan; \\ 2Department of Emergency Medicine, \\ ASST Grande Ospedale Metropolitano \\ Niguarda, Milan, Italy
}

\begin{abstract}
Acute abdominal pain represents a challenge for the physician because it can hide a serious intra-abdominal pathology necessitating emergency intervention. A 65-yearold man presented to Emergency Department with sudden-onset abdominal pain. He underwent liver transplantation four years before. He complained tenderness on abdominal palpation. Blood chemistry and abdominal $\mathrm{x}$-ray were normal. Contrast-enhanced computed tomography showed acute renal artery thrombosis. The patient underwent renal arterial thrombectomy and stent placement in less than two hours. Organ transplantation is a condition that makes patients at greater risk of lifethreatening conditions. Renal artery thrombosis is a rare, severe and misdiagnosed condition which can benefit from a prompt cooperation among emergency physician, surgeon, and interventional radiologist. Transplant patients with acute abdominal pain should be considered at high risk of medical emergency. Acute renal artery thrombosis is a time dependent medical emergency in those patients with chronic drug-induced nephrotoxicity.
\end{abstract}

\section{Introduction}

Acute abdominal pain is a common pathological condition ${ }^{1}$ and a frequent cause of access to Emergency Department. ${ }^{2}$ It could be categorized on the basis of the etiology in eight main diagnoses: appendicitis, cholecystitis, bowel obstruction, renal colic, peptic ulcer disease, pancreatitis, diverticular disease, and nonspecific abdominal pain. Less common causes of acute abdominal pain could be visceral perforation, vascular complications, such as abdominal aortic aneurysm rupture, mesenteric ischemia or thrombosis of others abdominal districts and pelvic disorders.
Acute abdominal pain is a challenge for the physician: it is often an expression of a benign condition, but in a minor percentage of patients it could represent a surgical emergency, ${ }^{3}$ that is dramatically important to identify as soon as possible to avoid a poor prognosis.

A collection of accurate patient's history, execution of physical examination, acquisition of proper laboratory test and appropriate radiological studies and, in some rare cases, the performance execution of diagnostic laparoscopy, represent the correct and the useful workup for these patients, to reduce the risk of missing diagnosis.

\section{Case Report}

A 65-year-old man presented to Emergency Department with abdominal pain localized in the lower abdominal quadrants, nausea, and retching. He denied alteration in bowel transit or other enteric disorders.

The patient medical history included: liver transplantation four years before for hepatocellular carcinoma (HCC) associated with alcoholic cirrhosis, diabetes mellitus, COPD, previous TB and surgical intervention for an abdominal hernia two years ago. His daily drugs were: tacrolimus, ursodeoxycholic acid, magnesium, and sodium sulfonated polystyrene. He denied any allergies.

Vital signs recorded at the admission were the following: blood pressure $165 / 80$ $\mathrm{mmHg}$, heart rate 54 beats per minute, pulse oximetry $98 \%$ on room air. The patient was apyretic. At first evaluation, the abdomen was soft, not distended, tender to palpation in the lower quadrants. Bowel sounds were present. Blumberg and Giordano's signs were negative. The rest of physical examination was normal. The first blood sample analysis showed no leukocytosis, CReactive Protein $1.4 \mathrm{mg} / \mathrm{dL}$ (normal range $<0.5$ ), normal haemoglobin, conserved renal function, lactate $2.78 \mathrm{Mmol} / \mathrm{L}$. ECG was normal. An abdominal x-ray was performed and showed only fecal impaction. The patient was treated with mild intravenous analgesic therapy, and three hours later during the surgeon visit, the pain was disappeared. About an hour after, the pain suddenly reappeared stronger than in the previous hours and not responsive to repeated morphine bolus.

Given the frailty of a transplanted patient and the importance of the pain without an identified cause, a contrast-enhanced computed tomography was performed. The images showed a strong reduction of con-
Correspondence: Paolo Aseni, Department of Emergency Medicine, ASST Grande Ospedale Metropolitano Niguarda, Milano, Italy. Tel.: +39.02644474433 - Fax: +39.0264442825.

Key words: acute abdominal pain; emergency department; renal artery thrombosis; liver transplantation.

Contributions: VB, PA, SDD, RC, MC, AB were all attending doctors for the patient during the Emergency evaluation and during the Hospital care. VB, PA organized the report and wrote the paper. $\mathrm{AB}, \mathrm{SDD}, \mathrm{MC}, \mathrm{RC}$ provided critical revision of the paper.

Conflict of interest: the authors declare no potential conflict of interest.

Received for publication: 31 July 2017. Accepted for publication: 13 October 2017.

This work is licensed under a Creative Commons Attribution 4.0 License (by-nc 4.0)

(C) Copyright V. Bozzano et al., 2017

Licensee PAGEPress, Italy

Emergency Care Journal 2017; 13:6966

doi:10.4081/ecj.2017.6966

trast-enhancement in the right kidney and a stenosis in the right renal artery strongly suggestive for renal artery acute thrombosis (Figure 1). The patient was immediately addressed to Interventional radiologist where renal arterial thrombectomy and stent placement were performed (Figure 2). At the end of the procedure contrast injection showed a good and homogenous perfusion of the right kidney. The time between the pain onset and the end of the procedure and between the radiological diagnosis and the end of the procedure was respectively 7 and 2 hours.

The patient was admitted in Surgery Ward, and he was discharged after four days without other complications and with a normal renal function.

\section{Discussion}

Transplantation is a condition that makes patients potentially at greater risk of dangerous conditions and complications than non-transplant population. In particular, patients who have received liver transplantation are more susceptible to biliary complications, such as biliary stricture, ischemia, presence of stone, vascular complications like stenosis or thrombosis of the hepatic artery, portal vein or hepatic vein and hepatic artery aneurysm, bowel 
obstruction, significant susceptibility to infections secondary to immunosuppressive therapy. ${ }^{4}$ For these reasons when transplant patients present abdominal symptoms, it would be appropriate to maintain a higher level of suspicion, because they often hide serious medical conditions.

Renal artery thrombosis is a rare and misdiagnosed condition. The prevalence is 2/100 000 in an Emergency Department population. ${ }^{5}$ Clinical presentation is insidious, and often patients present symptoms similar to many other abdominal conditions and not specific laboratory tests. The gold standard for the diagnosis is angiography, and the most commonly used method is contrast-enhanced computed tomography. ${ }^{6}$ Renal artery thrombosis could be associated with other pathologic conditions, ${ }^{5,7}$ but no links between liver transplantation and renal thrombosis have been found. We speculate that the long immunosuppressive therapy with Tacrolimus that transplanted patients have to assume could predispose to renal artery thrombosis, but no evidence has been found in literature. It should be highlighted that the importance of a proper diagnosis of thrombosis and the early revascularisation in this particular patient was very important to preserve his right kidney. Transplant patients receiving long-term immunosuppressive therapy for organ transplantation usually with calcineurin inhibitors are at high risk of progressive loss of renal function and impairment.

Finally, we want briefly speculate in this patient the possible role of cooperation between the emergency physician and surgeon in the evaluation of acute abdominal

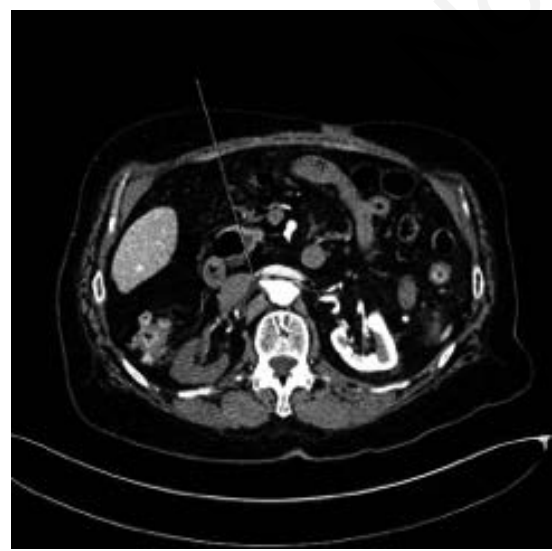

Figure 1. Abdominal contrast-enhanced computed tomography exhibiting a strong reduction of contrast-enhancement in the right kidney. The arrow represents the stenosis. pain. Despite all efforts, emergency physicians and surgeons are rarely sharing characteristics and joint responsibilities of emerging importance, and in the majority of hospitals, there is little collaboration between them. These two specialists do not routinely work together, missing opportunities to examine together medical and surgical evidence, develop protocols, design referral programs for admission, conduct quality reviews and co-manage difficult patients in the emergency setting. On the contrary, the real world of emergency setting seems to suggest sometimes that a strong cooperation between surgeons and emergency physicians can allow unexpected positive results in term of rapidity for a correct diagnosis and therapy. The importance of sharing quick decision in selected unstable or frail patients between emergency physicians and surgeons, should not be underestimated. This can allow a certain degree of improved collaboration which can be paramount in some particular emergency circumstances. It should be of interest to identify some areas in emergency medicine of mutual interest as opportunities for cooperation between emergency physicians and surgeons in order to verify possible benefits for improving the patient care.

\section{Conclusions}

Patients who underwent transplantation, particularly liver transplantation, are frail patients who are more susceptible to dangerous clinical complications; in these patients, the clinical suspicion should always be very high.

Renal artery thrombosis is a rare and misdiagnosed condition, but for its severity and its possible dramatic consequences, physician should consider it in the differential diagnosis of acute abdominal pain.
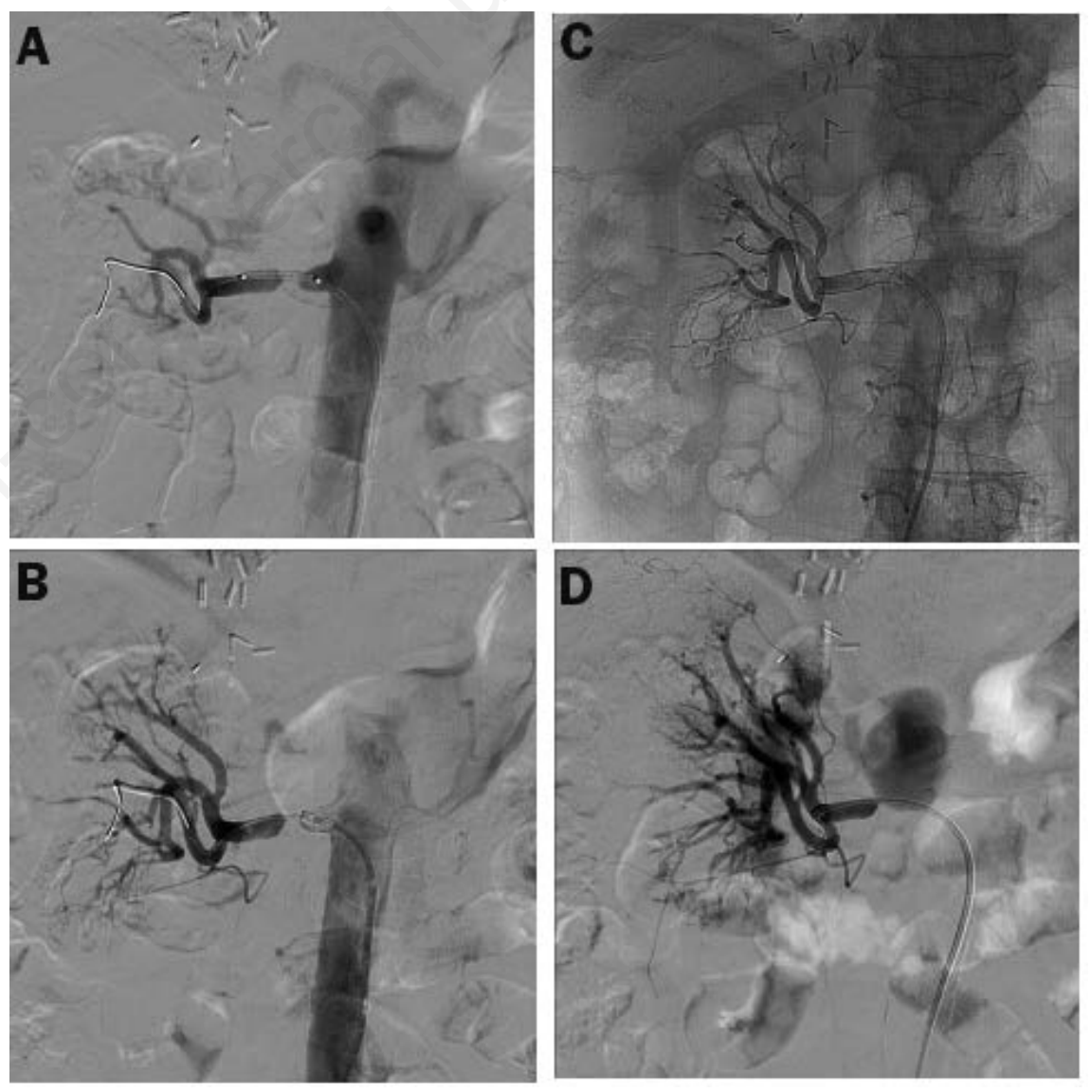

Figure 2. Stent placement during the angiography procedure. A) Right renal artery acute thrombosis; B) a guide wire is passed through the stenosis with the stent over the guide wire; C) delivery of the stent; D) homogenous kidney reperfusion showing a good runoff. 


\section{References}

1. Cartwright SL, Knudson MP. Evaluation of acute abdominal pain in adults. Am Fam Physician 2008;77:971-8.

2. Laurell H, Hansson LE, Gunnarsson U. Impact of clinical experience and diagnostic performance in patients with acute abdominal pain. Gastroent Res
Pract 2015;2015:590346.

3. Hardy A, Butler B, Crandall M. The evaluation of acute abdomen. In: Moore LJ, Turner KL, Todd SR, eds. Common problems in acute care surgery. Berlin: Springer-Verlag; 2013. pp 20-24.

4. Porrett PM, Hsu J, Shaked A. Late surgical complications following liver transplantation. Liver Transplant 2009;15: S12-8.

5. Raghavendran M, Sarkar M, Kumar
KG. Isolated spontaneous renal artery thrombosis. A rare cause of acute flank pain. Urol Case Rep 2016;9:4-5.

6. Lopez VM, Glauser J. A case of renal artery thrombosis with renal infarction. J Emerg Trauma Shock 2010;3:302-8.

7. Mesiano P, Rollino C, Beltrame $\mathrm{G}$ et al. Acute renal infarction: a single centre experience. J Nephrol 2017;30:1037 